Copyright (C) 2005 IEEE. Reprinted from

IEEE Conference on Decision and Control and European Control

Conference (44th : 2005 : Seville, Spain)

This material is posted here with permission of the IEEE. Such permission of the IEEE does not in any way imply IEEE endorsement of any of the University of Adelaide's products or services. Internal or personal use of this material is permitted. However, permission to reprint/republish this material for advertising or promotional purposes or for creating new collective works for resale or redistribution must be obtained from the IEEE by writing to pubs-permissions@ieee.org.

By choosing to view this document, you agree to all provisions of the copyright laws protecting it. 


\title{
Active control of structural sound radiation using a spatial control method with multiple structural sensors
}

\author{
Dunant Halim and Ben S. Cazzolato
}

\begin{abstract}
This paper introduces a method to control structural sound radiation using multiple structural sensors. Tonal radiation from a vibrating arbitrary structure is considered in this paper. Based on the estimation of the vibration profile of a noise radiating structure, spatial signals that represents radiated pressure field can be generated. These spatial signals can also be spatially weighted to control radiated noise at some far-field regions more than at others. Numerical studies on a simply-supported plate were performed which demonstrate the ability of the proposed method to control sound radiation that is spatially weighted over certain regions in the far-field.

Index Terms-sound radiation; structures; structural sensors; spatial signal; spatial weighting
\end{abstract}

\section{INTRODUCTION}

It is well known that a vibrating structure can radiate sound. Much research has been concentrated on attempting to control sound radiated from structures such as in [1], [2], [3], [4], in which structural sensors are used in some works. Meirovitch et al [5], [6] proposed the use of modal filtering to obtain information about the system's modal amplitudes from structural sensor measurements. Other works such as in [7], [3] used vibration information from multiple structural sensors for sound radiation control.

In some cases, it might be desirable to control sound radiation at specific regions/directions, allowing the control effort to be concentrated for control at those regions only. A spatially weighted objective can be used to target specific regions that are of importance, where sound pressure reduction is desired. Some researchers have proposed spatial control methods based on optimal control frameworks [8], [9], [10]. Spatially weighted objectives were incorporated in control design to target certain regions in spatially distributed systems, such as certain spatial regions in vibrating structures. However, the spatial control methods rely on the availability of the system's dynamic model, which might not be easy to obtain in practice.

Therefore, this work is devoted to the development of a spatial control strategy for structural sound radiation control. The proposed strategy can be used without apriori information of the structure's dynamics by using velocity measurements from multiple structural sensors. Although other works in structural vibration control ([5], [6], [11], [12], for instance) also utilised spatial interpolations, the

D. Halim (corresponding author) is with the School of Mechanical Engineering, University of Adelaide, SA 5005, Australia. Tel: (+61 8) 8303 6941, Fax: (+61 8) 8303 4367, Email: dunant.halim@adelaide.edu.au

Ben S. Cazzolato is with the School of Mechanical Engineering, University of Adelaide, SA 5005, Australia.Tel: (+61 8) 8303 5449, Fax: (+61 8) 8303 4367, Email: benjamin.cazzolato@adelaide.edu.au

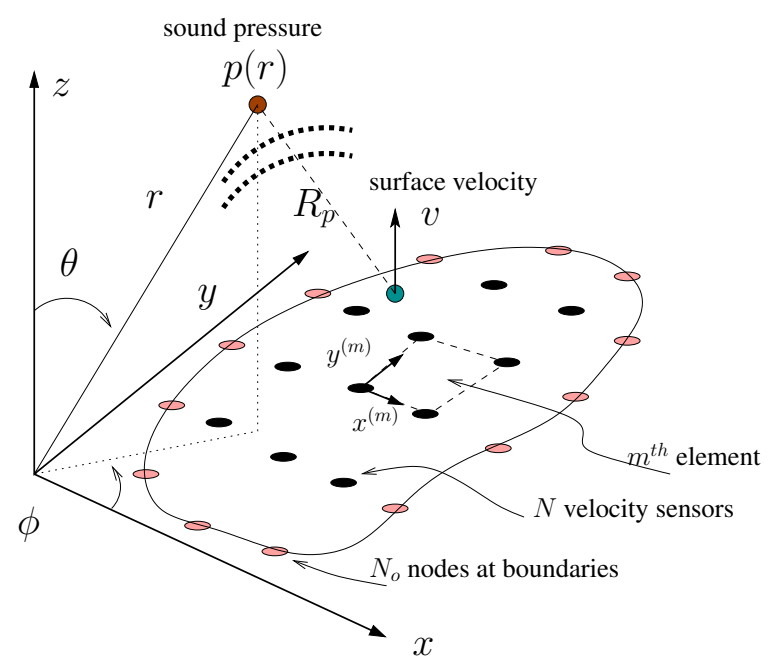

Fig. 1. A sound radiating structure with $N$ structural velocity sensors and $N_{o}$ nodes at boundaries. In this example, the $m^{\text {th }}$ element has 4 nodes, whose local coordinates are $\left(x^{(m)}, y^{(m)}\right)$.

previous works still require some apriori structural information such as modal or structural properties. Furthermore, a spatially weighted objective can be incorporated by employing a continuous spatial weighting that specifies the desired regions/directions for sound radiation reduction. Spatial interpolations based on the information from structural velocity sensors are used to estimate the velocity profile of a vibrating structure. This velocity profile measurements is then exploited to estimate the sound radiated from the structure for control purposes.

\section{SENSING OF STRUCTURAL SOUND RADiaton}

This section discusses the use of multiple structural velocity sensors to estimate the structural velocity profile that consequently relates to the noise radiated from a vibrating structure. In this case, the apriori information about the dynamics of the structure is not required, except for the geometric configuration of sensors on the structure.

\section{A. Vibration velocity sensing}

The velocity profile of a structure is estimated via a spatial interpolation method, incorporating the information from structural sensors [15]. The interpolation method resembles the finite element method commonly used for numerical analysis of structural dynamics, such as in [13], [14], [5].

Consider an arbitrary structure shown in Fig. 1, where $N$ structural velocity sensors are attached to the structure. 
The $i^{\text {th }}$ sensor measures normal velocity $v_{i}$ at a particular structural location $\left(x_{i}, y_{i}\right)$. Each sensor can be considered as a 'node' that will be used to construct 'elements' over the structure to estimate the structural vibration profile. To capture the entire structural vibration profile, additional nodes near the structural boundaries are also needed. In many cases, the level of vibration (velocity) at the boundaries will be practically minimal, so it is not necessary to place additional sensors at these regions. Instead, 'temporary' nodes are added to help construct the overall vibration profile of the structure. These 'temporary' nodes will be removed at a later stage of the sound radiation sensing process since the vibration levels at those nodes are minimal.

Suppose there are $N$ velocity sensors and $N_{o}$ 'temporary' nodes at structural boundaries. Then $M$ elements can be constructed from $N+N_{o}$ nodes over the structure. Next, consider the $m^{t h}$ element, whose local coordinates are $\left(x^{(m)}, y^{(m)}\right)$ as shown in Fig. 1. In this case, the velocity level $v_{x y}^{(m)}$ at a location $\left(x^{(m)}, y^{(m)}\right)$ can be spatially interpolated from velocity measurements by $l$ velocity sensors associated with this $m^{t h}$ element. For the example shown in Fig. $1, l=4$. Suppose that $\mathbf{v}^{(m)}$ is a set of $l$ velocity measurements $v_{i}$ associated with the $m^{t h}$ element, and $\mathbf{H}\left(x^{(m)}, y^{(m)}\right)$ is a $1 \times l$ interpolation function matrix. A linear transformation matrix $\mathbf{A}^{(m)} \in \mathbf{R}^{l \times\left(N+N_{o}\right)}$ is utilised to transform local coordinates $\left(x^{(m)}, y^{(m)}\right)$ to global coordinates $(x, y)$. The velocity profile of the structure can then be estimated by [15]

$$
v_{x y}(x, y)=\mathbf{H}\left(x^{(m)}, y^{(m)}\right) \mathbf{A}^{(m)} \mathbf{v} .
$$

Linear or higher-order polynomial interpolation functions commonly used in finite element method [13], [14] can be used for this interpolation purpose. This velocity profile measurement will be crucial in estimating the sound radiated from the structure as explained in the following.

\section{$B$. Sound radiation sensing}

Consider a planar structure, vibrating at frequency $\omega_{o}$, that radiates sound as depicted in Fig. 1. The sound pressure at a point of interest $p(r)$, a distance $r$ away from the coordinate system's origin, can be estimated by the Rayleigh integral [16]:

$$
p(r)=\int_{S} \frac{j \omega_{o} \rho_{o} v(S) e^{-j k R_{p}} d S}{2 \pi R_{p}}
$$

where $v$ is the normal surface velocity, $S$ is the radiating surface of the structure, $\rho_{o}$ is the density of acoustic medium, and $R_{p}$ is the distance from each structural point to the point of interest. Also, $k$ is the wave number, i.e. $k=2 \pi / \lambda$ where $\lambda$ is the wave-length of the radiated sound.

In this work, the far-field sound radiation case is considered, a situation many practical sound radiation problem can be approximated by. Here, the far-field assumption [16] is used where $R_{p}$ is much larger than the dimensions of the structure. Thus, $R_{p}$ in term $e^{-j k R_{p}}$ in (2) is approximated by $R_{p} \approx r-x \sin \theta \cos \phi-y \sin \theta \sin \phi$, while $R_{p}$ in the denominator is approximated by $r$. Based on the spherical coordinate system in Fig. 1, the sound pressure at far-field can be expressed as:

$$
\begin{aligned}
p(r, \theta, \phi)= & \frac{j \omega_{o} \rho_{o}}{2 \pi r} \int_{S} e^{-j k(r-x \sin \theta \cos \phi-y \sin \theta \sin \phi)} \\
& \times v(S) d S .
\end{aligned}
$$

The structural surface velocity $v$ can now be estimated by the surface velocity profile from sensor measurements $v_{x y}$ (1). The sound pressure can be found by considering the contributions of all $M$ elements:

$$
\begin{aligned}
p(r, \theta, \phi)= & \frac{j \omega_{o} \rho_{o} e^{-j k r}}{2 \pi r}\left\{\sum_{m=1}^{M} e^{j\left(\alpha x_{o}^{(m)}+\beta y_{o}^{(m)}\right)}\right. \\
& \times\left(\int_{x^{(m)}} \int_{y^{(m)}} \mathbf{H}\left(x^{(m)}, y^{(m)}\right) e^{j \alpha x^{(m)}}\right. \\
& \left.\left.\times e^{j \beta y^{(m)}} d x^{(m)} d y^{(m)}\right) \mathbf{A}^{(m)}\right\}_{\mathbf{v}}
\end{aligned}
$$

where

$$
\begin{aligned}
& \alpha=k \sin \theta \cos \phi \\
& \beta=k \sin \theta \sin \phi
\end{aligned}
$$

and $\left(x_{o}^{(m)}, y_{o}^{(m)}\right)$ is the origin's location for the $m^{t h}$ element.

\section{Spatially weighted cost function}

Having estimated the sound pressure from the structure, the objective in this work is to control sound radiation in some far-field regions of interest. In this case, a spatially weighted cost function is used which allows a spatial filtering to be used for active control purposes.

Consider the case where some far-field regions $(\theta, \phi)$ have more importance than others. A scalar continuous spatial weighting $q(\theta, \phi)$ is introduced where $q(\theta, \phi) \geq 0 \forall \theta \in$ $[0, \pi / 2]$ and $\phi \in[0,2 \pi]$. The following cost function is used:

$$
J_{p}=\int_{0}^{2 \pi} \int_{0}^{\frac{\pi}{2}} p(r, \theta, \phi)^{H} q(\theta, \phi) p(r, \theta, \phi) d \theta d \phi
$$

where $(F)^{H}$ is the Hermitian transpose of a matrix $F$.

Substituting (4) into $J_{p}$ in (6), the following can be obtained:

$$
\begin{aligned}
J_{p}= & \mathbf{v}^{H}\left\{\frac{\omega_{o} \rho_{o}}{2 \pi r}\right\}^{2}\left\{\int _ { 0 } ^ { 2 \pi } \int _ { 0 } ^ { \frac { \pi } { 2 } } \left(\sum_{m=1}^{M} \mathbf{A}^{(m) T} \mathbf{M}^{(m) H}\right.\right. \\
& \left.\times B^{(m) H}\right)_{q(\theta, \phi)}\left(\sum_{p=1}^{M} B^{(p)} \mathbf{M}^{(p)} \mathbf{A}^{(p)}\right) \\
& \times d \theta d \phi\} \mathbf{v} \\
= & \mathbf{v}^{H} \mathbf{P} \mathbf{v}
\end{aligned}
$$

where

$$
\begin{aligned}
B^{(m)}= & e^{j\left(\alpha x_{o}^{(m)}+\beta y_{o}^{(m)}\right)} \\
\mathbf{M}^{(m)}= & \int_{x^{(m)}} \int_{y^{(m)}} \mathbf{H}\left(x^{(m)}, y^{(m)}\right) e^{j\left(\alpha x^{(m)}+\beta y^{(m)}\right)} \\
& \times d x^{(m)} d y^{(m)}
\end{aligned}
$$


The integral term in (7) can be solved numerically and it can be shown that $\mathbf{P}$ is Hermitian. Furthermore, the integrand in (7) is generally positive semi-definite for every $(\theta, \phi)$ combination where $\theta \in[0, \pi / 2]$ and $\phi \in[0,2 \pi]$. However, since the term is integrated over the entire $(\theta, \phi)$ region, it can be shown that $\mathbf{P}$ is positive definite in general.

At this stage, since it is known that the vibration level is minimal at $N_{o}$ 'temporary' nodes, $\mathbf{P}$ can be condensed by removing the appropriate rows and columns that correspond to the associated nodes, reducing the dimensions of $\mathbf{P}$ from $N+N_{o}$ to $N$.

\section{Spatial filter matrix $\mathbf{\Pi}$}

Next, $\mathbf{P}$ in (7) can be decomposed as follows:

$$
\begin{aligned}
\mathbf{P} & =\boldsymbol{\Pi}^{H} \boldsymbol{\Pi} \\
& =\mathbf{U V U}^{H}
\end{aligned}
$$

where $\mathbf{V}$ is a diagonal matrix containing positive real valued eigenvalues because $\mathbf{P}>0$. Furthermore, $\mathbf{U}$ is a unitary matrix $\left(\mathbf{U} \mathbf{U}^{H}=\mathbf{I}\right)$ containing the associated eigenvectors. Matrix $\Pi$ is calculated from

$$
\boldsymbol{\Pi}=\mathbf{V}^{1 / 2} \mathbf{U}^{H} .
$$

Thus, the cost function $J_{p}$ in (7) can be simply written as:

$$
\begin{aligned}
J_{p} & =\mathbf{v}^{H} \boldsymbol{\Pi}^{H} \boldsymbol{\Pi} \mathbf{v} \\
& =\mathbf{v}_{\text {noise }}^{H} \mathbf{v}_{\text {noise }}
\end{aligned}
$$

where $\mathbf{v}_{\text {noise }}=\boldsymbol{\Pi} \mathbf{v}$ are the spatial signals that represent the spatially weighted noise radiation cost function in the far-field. The significance of this representation is that the spatial signals $\mathbf{v}_{\text {noise }}$ can be used as error signals for active control purposes as will be explained later in this section.

Since the estimate of sound radiation is based on velocity profile measurements from a finite number of structural sensors, it is obvious that a sufficient number of sensors are required for accurate sound radiation estimation in the far-field. However, the larger the number of sensors used, the greater would be the dimensions of the spatial signals $\mathbf{v}_{\text {noise }}$ that are used as the error signals. Consequently, the active control algorithm would be computationally more demanding, which may not be practical since large multichannel control inputs are required. Therefore, it is desirable to reduce the number of spatial signals so the number of inputs into the controller can also be reduced for practical implementation.

Based on the information about the eigenvalues of $\boldsymbol{\Pi}$ in (9), the relative importance of each eigenmode can be compared. Choosing the $N_{l}$ largest eigenvalues, the spatial filter matrix can be estimated by:

$$
\tilde{\boldsymbol{\Pi}}^{H} \tilde{\boldsymbol{\Pi}}=\sum_{i=1}^{N_{l}} \lambda_{i} \mathbf{u}_{i} \mathbf{u}_{i}^{H}
$$

where $\lambda_{i}$ and $\mathbf{u}_{i}$ are the $i^{\text {th }}$ largest eigenvalue and the associated eigenvector respectively; $N_{l}<N$ and $N$ is the number of sensors used.
Fig. 2 illustrates the use of spatial filtering for sound radiation control. The velocity measurements from the sensors are filtered by $\tilde{\boldsymbol{\Pi}}$ to produce condensed spatial signals $\mathbf{v}_{\text {noise }}$. These spatial signals serve as the error signals that can be minimised by various standard active control algorithms such as adaptive algorithms [17]. Control actuators are then used to generate forces to control structural vibration in a way that reduces sound radiation at some desired far-field regions.

\section{OPTIMAL SOUND RADIATION CONTROL WITH SPATIAL FILTERING}

Consider primary disturbances $\mathbf{d}$ at frequency $\omega_{o}$, that generate vibration on a structure. The velocity levels $\mathbf{v}$ at $N$ structural sensors attached can be found from

$$
\begin{aligned}
\mathbf{v}\left(j \omega_{o}\right)= & \mathbf{G}_{v d}\left(j \omega_{o}\right) \mathbf{d}\left(j \omega_{o}\right)+\mathbf{G}_{v w}\left(j \omega_{o}\right) \\
& \times \mathbf{w}\left(j \omega_{o}\right)
\end{aligned}
$$

where $\mathbf{w}$ are the control signals, and $\mathbf{G}_{j i}$ represents the transfer matrix from $\mathbf{i}$ to $\mathbf{j}$.

The spatial signals from (11), omitting $\left(j \omega_{0}\right)$ term, are

$$
\mathbf{v}_{n o i s e}=\tilde{\boldsymbol{\Pi}} \mathbf{G}_{v d} \mathbf{d}+\tilde{\boldsymbol{\Pi}} \mathbf{G}_{v w} \mathbf{w}
$$

where the condensed spatial filtering $\tilde{\Pi}$ has been used here, instead of $\boldsymbol{\Pi}$.

Using a quadratic minimisation approach [17], the minimum cost function $J_{p}^{\min }$ is

$$
\begin{aligned}
J_{p}^{\min }= & \mathbf{d}^{H} \mathbf{G}_{v d}^{H} \tilde{\boldsymbol{\Pi}}^{H} \tilde{\boldsymbol{\Pi}} \mathbf{G}_{v d} \mathbf{d}+\left(\mathbf{d}^{H} \mathbf{G}_{v d}^{H} \tilde{\boldsymbol{\Pi}}^{H}\right) \\
& \times \tilde{\boldsymbol{\Pi}} \mathbf{G}_{v w} \mathbf{w}_{o p t}
\end{aligned}
$$

where the optimal control input $\mathbf{w}_{\text {opt }}$ is

$$
\begin{aligned}
\mathbf{w}_{o p t}= & -\left(\mathbf{G}_{v w}^{H} \tilde{\boldsymbol{\Pi}}^{H} \tilde{\boldsymbol{\Pi}} \mathbf{G}_{v w}\right)^{-1} \times \mathbf{G}_{v w}^{H} \tilde{\boldsymbol{\Pi}}^{H} \\
& \times \tilde{\boldsymbol{\Pi}} \mathbf{G}_{v d} \mathbf{d} .
\end{aligned}
$$

\section{NUMERICAL STUDIES: SOUND RADIATION CONTROL OF A PLATE}

This section describes how the proposed method can be used for optimal control of structural sound radiation. For this purpose, a simply-supported rectangular plate was used since there exists an analytical solution for the far-field sound radiation [18], so the sound pressure estimation can be readily compared. The plate's size is $800 \mathrm{~mm} \times 600 \mathrm{~mm} \times$ $4 \mathrm{~mm}$ with $N$ velocity sensors attached across the plate as illustrated in Fig. 3 (see Table I for properties of the plate). Here, $N=25$ is used with point forces as the primary disturbance and control sources. The modal analysis method is used for modelling of the plate [19], considering the first 24 modes, up to $800 \mathrm{~Hz}$. Table II lists the first 4 resonance frequencies of the plate.

In this study, the spatial interpolation functions used are linear functions where rectangular elements are utilised. The size of each element is $h_{x}^{(m)} \times h_{y}^{(m)}$, with $l=4$ velocity sensors (at all corners of the element) are used as the nodes (see Fig. 3). The coordinates of each element are $x^{(m)}$ and 


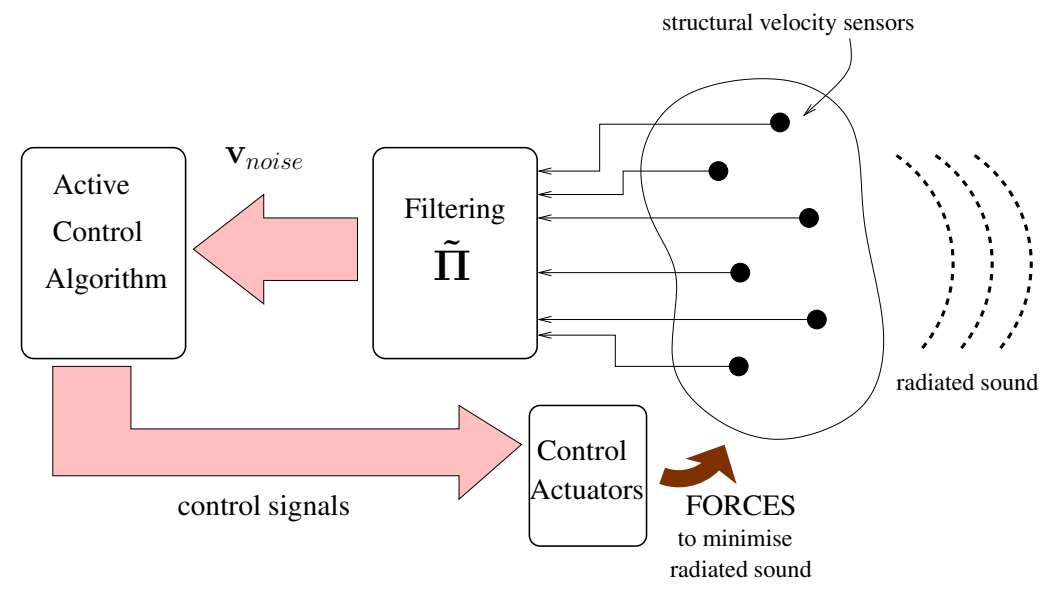

Fig. 2. A general approach for sensing and control using spatial signals

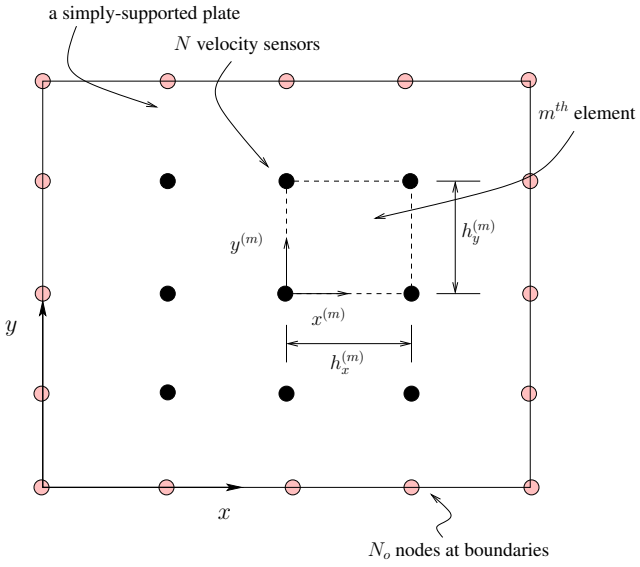

Fig. 3. A simply-supported plate with structural velocity sensors

TABLE I

PROPERTIES OF THE SIMPLY-SUPPORTED PLATE

\begin{tabular}{ll}
\hline Plate Young's Modulus & $7.0 \times 10^{10} \mathrm{~N} / \mathrm{m}^{2}$ \\
Plate Poisson's ratio & 0.30 \\
Plate density & $2750 \mathrm{~kg} / \mathrm{m}^{3}$ \\
\hline
\end{tabular}

$y^{(m)}$ and the linear interpolation function matrix $\mathbf{H}$ described in (1) is

$$
\mathbf{H}=\left[\begin{array}{c}
\left\{1-x_{h}^{(m)}\right\}\left\{1-y_{h}^{(m)}\right\} \\
x_{h}^{(m)}\left\{1-y_{h}^{(m)}\right\} \\
\left\{1-x_{h}^{(m)}\right\} y_{h}^{(m)} \\
x_{h}^{(m)} y_{h}^{(m)}
\end{array}\right]^{T}
$$

where

$$
\begin{aligned}
x_{h}^{(m)} & =\frac{x^{(m)}}{h_{x}^{(m)}} \\
y_{h}^{(m)} & =\frac{y^{(m)}}{h_{y}^{(m)}} .
\end{aligned}
$$

TABLE II

RESONANCE FREQUENCIES OF THE FIRST FOUR MODES OF THE PLATE

\begin{tabular}{lll}
\hline No. & Mode & Frequency $(\mathrm{Hz})$ \\
\hline 1 & $(1,1)$ & 41.6 \\
2 & $(2,1)$ & 86.6 \\
3 & $(1,2)$ & 121.6 \\
4 & $(3,1)$ & 161.5 \\
\hline
\end{tabular}

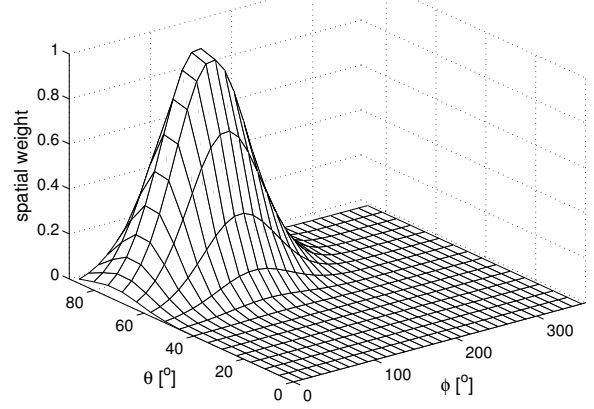

Fig. 4. The spatial weighting function, emphasising the angular regions where sound reduction is more important.

To demonstrate the control performance in reducing the spatially weighted sound radiation in the far-field, the following numerical studies are performed.

In the first numerical study, point forces are used for a primary disturbance source and two secondary (control) sources whose locations are $(0.229 \mathrm{~m}, 0.214 \mathrm{~m})$ and $(0.308 \mathrm{~m}, 0.429 \mathrm{~m}),(0.570 \mathrm{~m}, 0.130 \mathrm{~m})$ respectively. Fig. 4 depicts the spatial weighting $q(\theta, \phi)$ used which reflects $(\theta, \phi)$ regions that are of importance. The objective is to minimise the sound radiated from the vibrating structure, giving more emphasis to the regions that have larger weights.

A condensation procedure is used to reduce the size of the spatial signal since the original size of the signal would require 25 control input channels. The 8 largest eigenvalues of $\mathbf{P}$ are plotted in Fig. 5. The figure shows that the 


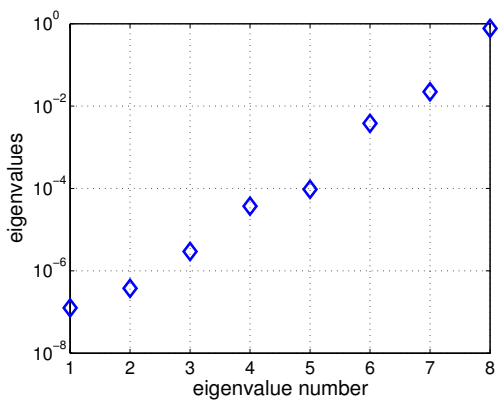

Fig. 5. The plot of 8 largest eigenvalues of $\mathbf{P}$. Four largest eigenvalues were used for reducing the size of the spatial filter.

eigenvalues vary significantly and it is thus reasonable to consider only several dominant eigenvalues. It was decided to use the 4 largest eigenvalues to generate a condensed spatial signal, which results in 4 control input channels.

Fig. 6 shows the sound pressure profile at $r=10 \mathrm{~m}$ for control at the resonance frequency of mode $(1,2)$, i.e. 121.6 Hz. The spatial sound radiation profile of uncontrolled system is shown in Fig. 6(a). Figs. 6(b) and (c) compare the spatial control performances using 1 and 2 control sources respectively. It is clear that the spatial control attempts to reduce the sound radiation more in the $(\theta, \phi)$ region of interest as reflected by the spatial weighting in Fig. 4.

The second numerical study concentrates on the control at the resonance frequency of mode $(3,1)(161.5 \mathrm{~Hz})$, using the same spatial weighting (Fig. 4). The simulation results in Fig. 7 demonstrate the effect of spatial control in reducing sound radiation at the specific angular $(\theta, \phi)$ region, although reduction is also achieved at other less-weighted regions. Figs. 7(b) and (c) clearly compare the effect of adding control sources to the control performance. When 2 control sources are used, the controller attempts to reduce sound pressure at the heavily-weighted $(\theta, \phi)$ region more, at the expense of increasing sound pressure at other regions. This is expected since the spatial control gives more emphasis to reducing the sound radiation in the regions that are more heavily weighted.

\section{COnClusions}

A spatial control method for controlling structural sound radiation using multiple structural sensors has been proposed. The sound radiation at far-field was estimated from the velocity profile of a vibrating arbitrary structure and a spatially weighted cost function was incorporated in the sensing procedure. Spatial signals representing the spatially weighted sound radiation in the far-field can be used as error signals that need to be minimised by standard active control algorithms. Numerical studies on a simply-supported plate described the benefit of the proposed method in controlling sound radiation at some regions in the far-field.

\section{ACKNOWLEDGEMENTS}

The authors are grateful for the financial support provided by the Australian Research Council (ARC), especially through the ARC PostDoctoral Research Fellowship for the first author.

\section{REFERENCES}

[1] E. K. Dimitriadis and C. R. Fuller, "Active control of sound transmission through elastic plates using piezoelectric actuators," American Institute of Aeronautics and Astronautics Journal, vol. 29, no. 11, pp. 1771-1777, 1992

[2] R. L. Clark and C. R. Fuller, "Control of sound radiation with adaptive structures," Journal of Intelligent Material Systems and Structures, vol. 2, pp. 431-452, 1991.

[3] N. C. Burgan, S. D. Snyder, N. Tanaka, and A. Z. Zander, "A generalised approach to modal filtering for active noise control - Part I: Vibration sensing," IEEE Sensors Journal, vol. 2, no. 6, pp. 577-589, 2002.

[4] S. G. Hill, S. D. Snyder, B. S. Cazzolato, N. Tanaka, and R. Fukuda, "A generalised approach to modal filtering for active noise control Part II: Acoustic sensing," IEEE Sensors Journal, vol. 2, no. 6, pp. 590-596, 2002.

[5] L. Meirovitch and H. Baruh, "Control of self-adjoint distributedparameter systems," Journal of Guidance, vol. 5, no. 1, pp. 60-66, 1982.

[6] _ " "The implementation of modal filters for control of structures," Journal of Guidance, vol. 8, no. 6, pp. 707-716, 1985.

[7] A. P. Berkhoff, "Sensor scheme design for active structural acoustic control," Journal of the Acoustical Society of America, vol. 108, no. 3 , pp. $1037-1045,2000$.

[8] S. O. R. Moheimani, H. R. Pota, and I. R. Petersen, "Spatial control for active vibration control of piezoelectric laminates," in Proc. of 37 th IEEE CDC, Tampa, Florida, December 1998, pp. 4308-4313.

[9] S. O. R. Moheimani, I. R. Petersen, and H. R. Pota, "Broadband disturbance attenuation over an entire beam," Journal of Sound and Vibration, vol. 227, no. 4, pp. 807-832, 1999.

[10] S. O. R. Moheimani, D. Halim, and A. J. Fleming, Spatial Control of Vibration: Theory and Experiments. Singapore: World Scientific, 2003

[11] G. A. Pajunen, P. S. Neelakanta, M. Gopinathan, and M. Arockaisamy, "Distributed adaptive control of flexible structures," SPIE Proc. 1994 North American Conf. on Smart Structures and Intelligent Systems, pp. 2190:790-801, 1994.

[12] M. Gopinathan, G. A. Pajunen, P. S. Neelakanta, and M. Arockaisamy, "Recursive estimation of displacement and velocity in a cantilever beam using a measured set of distributed strain data," Journal of intelligent material systems and structures, vol. 6, pp. 537-549, 1995.

[13] D. Halim and B. S. Cazzolato, "A multiple-sensor method for control of structural vibration with spatial objectives," Journal of Sound and Vibration, 2005, submitted.

[14] K. J. Bathe and E. L. Wilson, Numerical Methods in Finite Element Analysis. Englewood Cliffs, New Jersey: Prentice Hall, 1976.

[15] Y. K. Cheung and A. Y. T. Leung, Finite Element Methods in Dynamics. Beijing, New York; Dordrecht, Boston: Science Press; Kluwer Academic Publishers, 1991.

[16] C. R. Fuller, S. J. Elliot, and P. A. Nelson, Active Control of Vibration. London: Academic Press, 1996.

[17] S. J. Elliot, Signal Processing for Active Control. Academic Press, 2001

[18] C. E. Wallace, "Radiation resistance of a rectangular panel," Journal of the Acoustical Society of America, vol. 51, pp. 946-952, 1972.

[19] C. W. de Silva, Vibration: Fundamentals and Practice. Boca Raton: CRC Press, 2000 


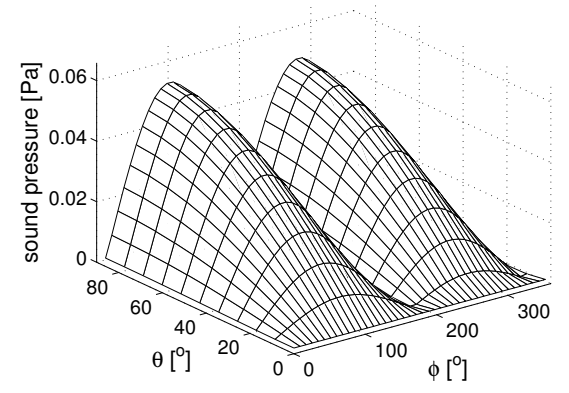

(a) No control - spatial pressure profile of mode $(1,2)$

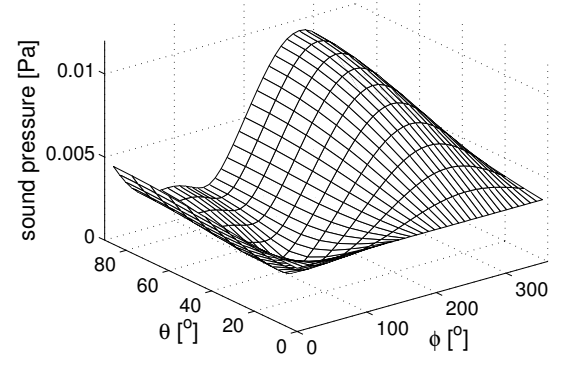

(b) 1 control source

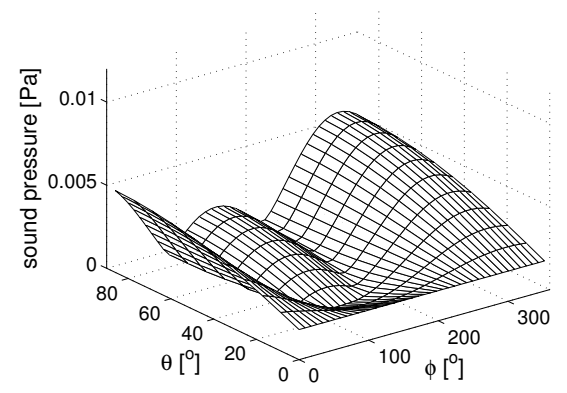

(c) 2 control sources

Fig. 6. Comparison of spatial control for mode $(1,2)$ using 1 control and 2 control sources.

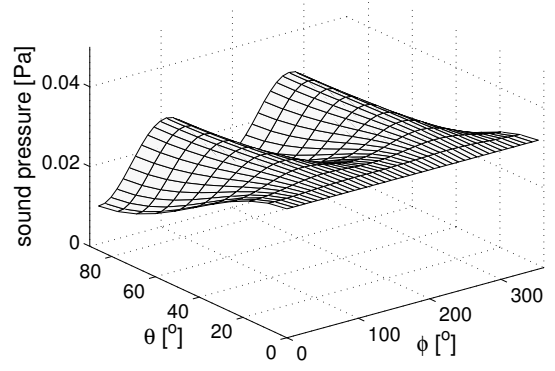

(a) No control - spatial pressure profile of mode $(3,1)$

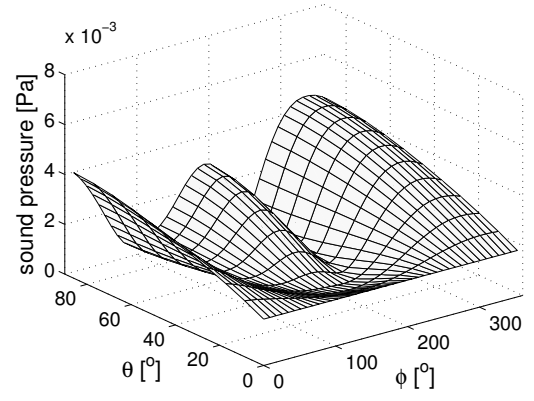

(b) 1 control source

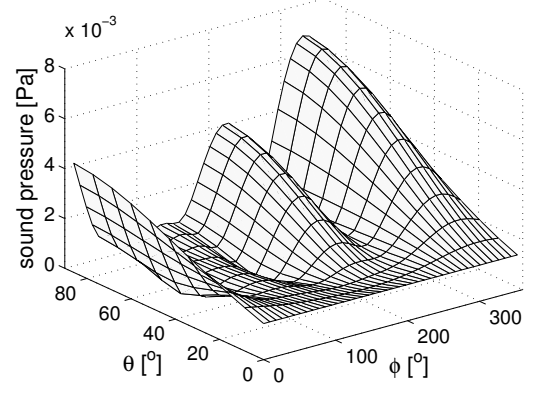

(c) 2 control sources

Fig. 7. Comparison of spatial control for mode $(3,1)$ using 1 control and 2 control sources. 